\title{
Nephritogenic Antibodies in MRL/1pr Lupus Mice: Molecular Characteristics in Pathological and Genetic Aspects
}

\author{
Junpei Itoh ${ }^{*} \dagger$, Satoru Takahashi $*$, Masao Ono* $\neq$, \\ Tokuo Yамамото $\$$, Masato Nose* and Masahisa \\ KYOGOKU* \\ *Department of Pathology, Tohoku University School of \\ Medicine, Sendai 980-7\%, †Laboratory of Biology, \\ Pathology Section, Nippon Kayaku Co., Tokyo 115, and \\ $\ddagger$ Gene Research Center, Tohoku University, Sendai 980-77
}

Itoh, J., Takahashi, S., Ono, M., Yamamoto, T., Nose, M. and Kyogoku, M. Nephritogenic Antibodies in MRL/lpr Lupus Mice: Molecular Characteristics in Pathological and Genetic Aspects. Tohoku J. Exp. Med., 1994, 173 (1), 65-74MRL/lpr mice spontaneously develop a lethal glomerulonephritis (GN). We found that IgG3 production in this strain of mice has a critical role on the development of GN; 1) IgG3 levels were high in kidney-extracted IgG and in circulating IgG immune complexes (IC), 2) serum IgG3 was selectively reduced by cyclosporin A treatment, associated with amelioration of GN, and 3) the mRNA levels of IgG3 correlated well with the severity of GN among the MRL/lpr $\times$ $(\mathrm{MRL} / \mathrm{lpr} \times \mathrm{C} 3 \mathrm{H} / \mathrm{lpr}) \mathrm{F}_{1}$ backcross mice with the rearranged genetic profile. Besed on these results, we have successfully established five hybridoma clones which produce nephritogenic IgG3 antibodies from an unmanipulated MRL/lpr mouse. When they were injected to normal mice, four of the five clones generated cell-proliferative GN associated with the marked cellular infiltrates, while the remaining clone induced wire loop-like lesions. This result suggests that particular antibodies generated in MRL/lpr mice have a different pathogenic potency. The V-region sequence study of these nephritogenic antibodies revealed that the two types of the glomerular lesions were mediated by a different $\mathrm{B}$ cell precursor. In conclusion, GN in MRL/lpr lupus mice is thought to be generated by the expansion of clonally different $\mathrm{B}$ cells producing nephritogenic antibodies with a different pathogenic potency.— $\longrightarrow$ MRL/lpr mice; lupus nephritis; IgG3; nephritogenic antibodies

MRL/Mp-lpr/lpr (MRL/lpr) mice have been used as an animal model for autoimmune diseases (Theofilopoulos and Dixon 1985; Itoh et al. 1991). They spontaneously develop a lethal glomerulonephritis (GN) accompanied by diffuse cell-proliferative and/or wire-loop lesions similar to those in human lupus nephritis (Andrews et al. 1978). These mice generate circulating immune complexes

Address for reprints: J. Itoh, Laboratory of Biology, Pathology Section, Nippon Kayaku Co., Ltd., 31-12 Shimo 3-chome, Kita-ku, Tokyo 115, Japan. 
(IC) associated with anti-DNA antibodies, rheumatoid factors, and/or anti-gp70 antibodies (Izui et al. 1979; Theofilopoulos and Dixon 1985). Close causative relationship between such serum components and development of GN has been postulated, of which reason has not been fully explained.

In this study, a series of experiments were undertaken to determine factor(s) crucial for the development of GN in MRL/lpr strain of mice. We examined the relationship between production of a particular IgG subclass and development of GN in MRL/lpr mice, and found that IgG3 production is a major factor responsible for the development of GN in these mice. This finding suggested the possible presence of nephritogenic IgG3 antibody-producing B cells in this strain of mice. Using an in vivo screening method, we successfully established five B cell hybridoma clones which produce nephritogenic IgG3 antibodies from an unmanipulated MRL/lpr mouse. Our result may shed a light on the generation of nephritogenic antibodies and the genetic basis of GN in this strain of mice.

IgG3 production is responsible for development of $G N$ in $M R L / l p r$ mice

IgG3 is the major subclass in kidney-extracted IgG and in circulating IgG IC. A particular subclass of IgG is associated with development of GN both in humans and in animals (Roberts et al. 1983; Gavalchin and Datta 1987). Based on these findings, a positive relationship between the production of a particular IgG subclass and the development of GN in MRL/lpr mice was expected.

A comparative study of IgG subclasses both in sera and kidney eluates from

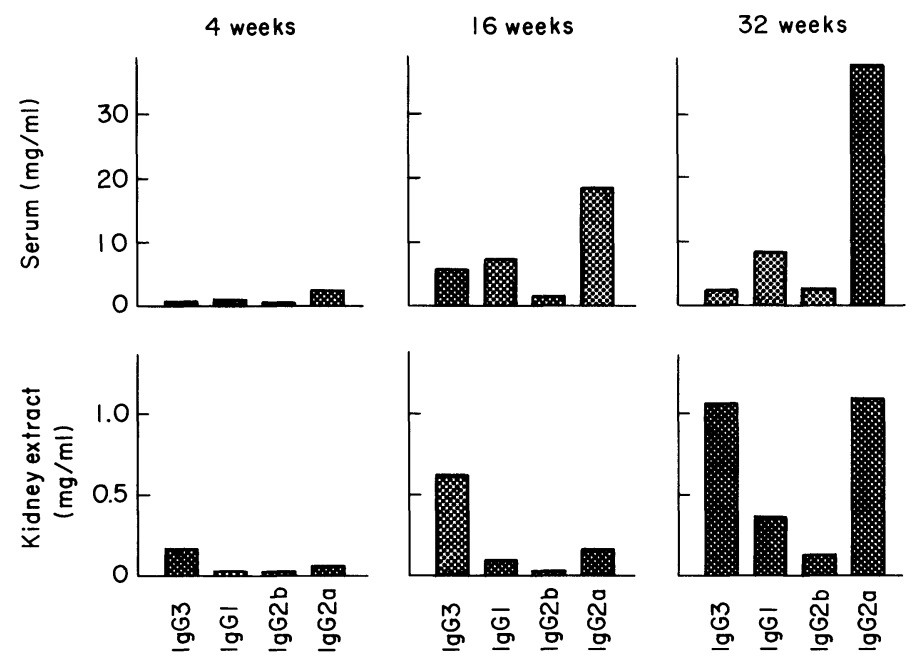

Fig. 1. Relative amounts of IgG subclasses in pooled sera and kidney-eluates of MRL/lpr mice (Takahashi et al. 1991). Pooled sera and kidneys from four mice of each age were used. The amount of each IgG subclass in the sample was measured by a single radial immunodiffusion assay. Each bar represents the mean of duplicate samples. 


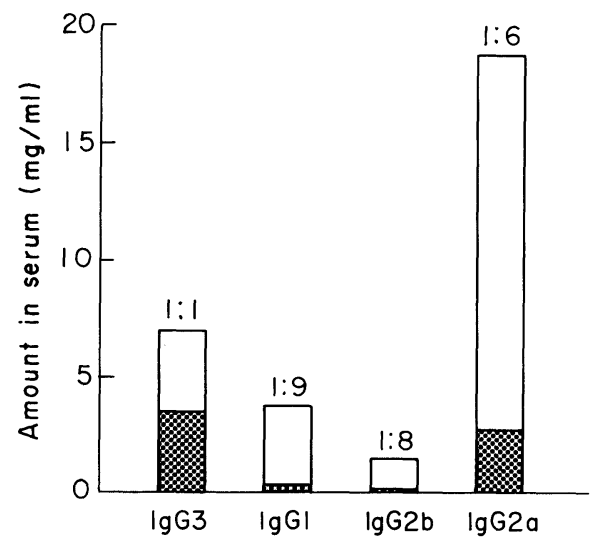

Fig. 2. Quantitative ratio of the amount of PEG-precipitates to the total amount of each IgG subclass in MRL/lpr mouse sera (Takahashi et al. 1991). Pooled sera from four 12-wk-old mice were used. The amount of each IgG subclass in the samples was measured by a single radial immunodiffusion assay. Each bar represents the mean of duplicate samples. Open bars, total amounts; stippled bars, amounts of PEG precipitates.

A

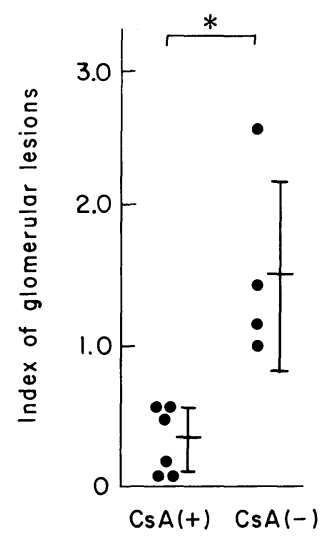

B

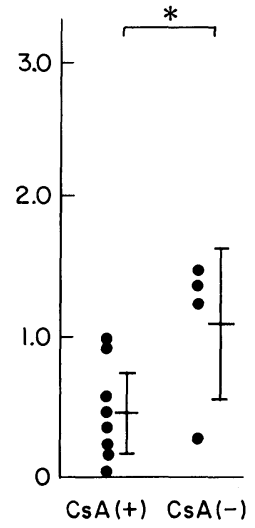

C

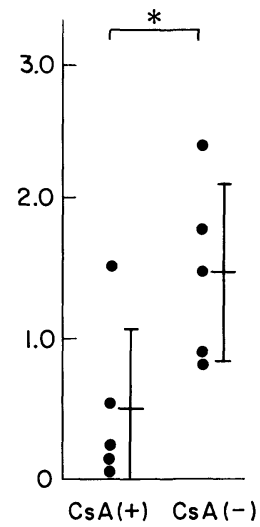

Fig. 3. Effect of CsA treatment on severity of glomerular lesions in MRL/lpr mice (Takahashi et al. 1991). CsA was dissolved in ethanol, diluted with olive oil, and orally administered to female MRL/lpr mice 6 days/wk from 6 to 17 (A), 12 to 20 (B), and 17 to $21 \mathrm{wk}$ of age (C), at a daily dose of $50 \mathrm{mg} / \mathrm{kg}$. Control mice were given only olive oil with ethanol. Mice were examined $1 \mathrm{wk}$ after the last administration. Each point represents the index of glomerular lesions of each mouse. Each bar represents the mean \pm s.D. *Significance in the difference between treated and untreated mice: $p<0.05$. 
MRL/lpr mice led to the following evidence: In sera, IgG2a was dominant in MRL/lpr mice at any age (Fig. 1, upper). All subclasses seemed to increase with age except IgG3 that decreased in 32-wk-old mice. In contrast, in kidney eluates, IgG3 was higher than any other subclass in 4-and 16-wk-old mice (Fig. 1, lower). Of the other IgG subclasses, IgG2a attained the same levels as IgG3 in 32-wk-old mice. Furthermore, it became clear that, in serum, IgG3 subclass is predominantly in an IC form (Fig. 2). In 12-week-old mice, almost 50\% of IgG3 in serum was in an IC form. IgG2a in an IC form was only $17 \%$ of the total amounts, although

$\lg$ I3

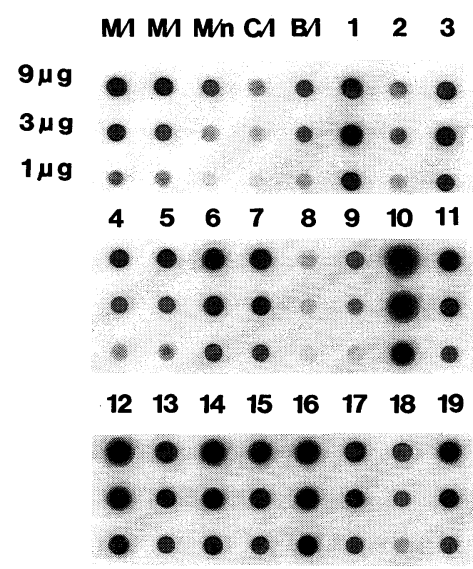

$\begin{array}{lllllll}20 & 21 & 22 & 23 & 24 & 25 & 26\end{array}$

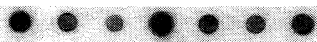

- $40 \%$ o

e $4=0$
IgG2a

A
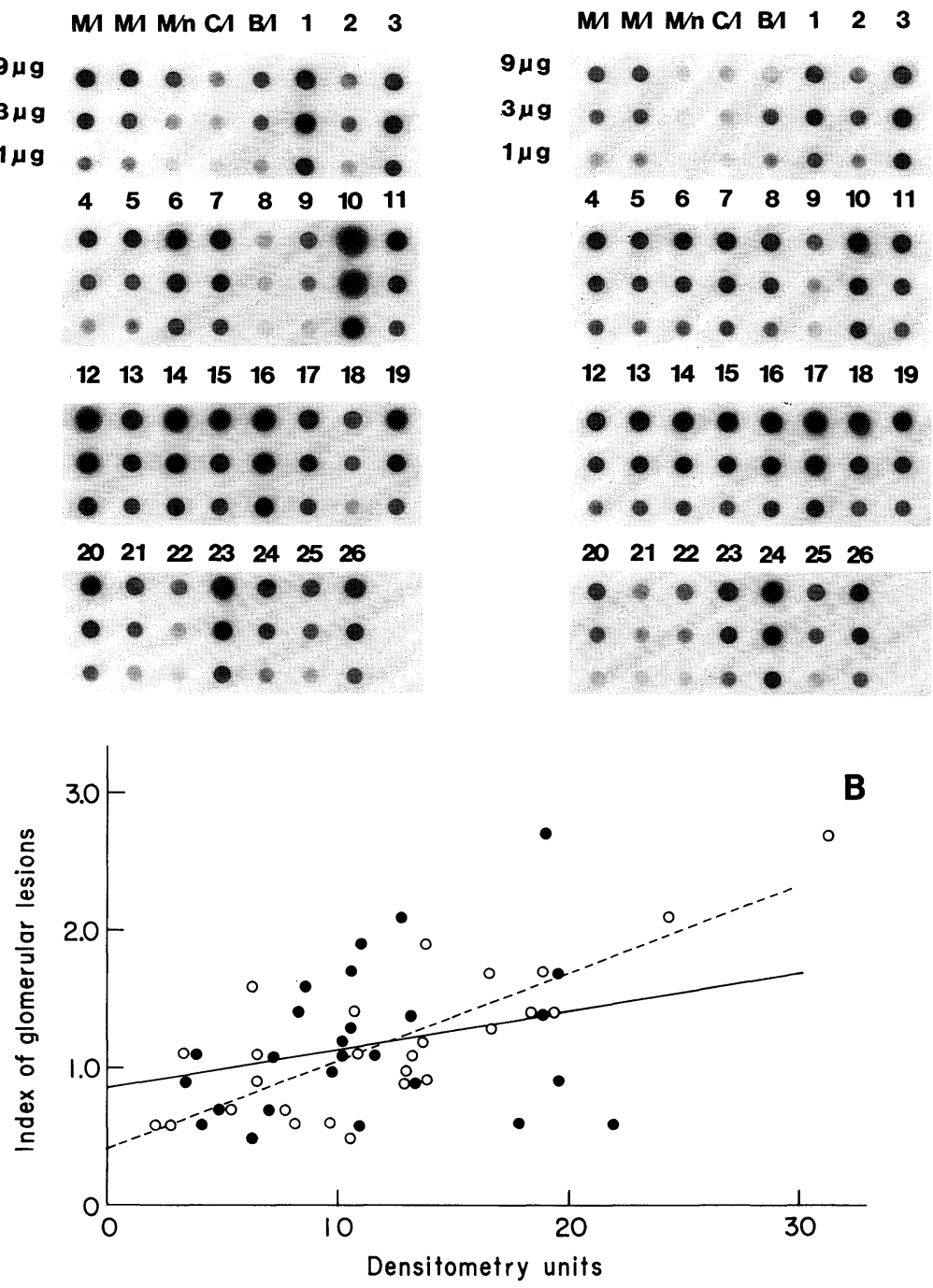

Fig. 4. 
the amounts of both IgG3 and IgG2a IC were almost equal.

Cyclosporin $A$ ameliorates $G N$ with reduced serum IgG3. Cyclosporin A (CsA) ameliorates GN in MRL/lpr mice, despite the ineffectiveness on autoantibody production (Mounts et al. 1987). To investigate this contradictory finding, the effect of CsA on IgG subclass levels in the sera of CsA-treated MRL/ lpr mice was studied. CsA treatment of MRL/lpr mice not only prevented but actually ameliorated their glomerular lesions (Fig. 3). A marked reduction of serum IgG3 was induced by CsA treatment in MRL/lpr mice, while none of the mice exhibited reduced autoantibody production or circulating IC in all groups treated with CsA (Takahashi et al. 1991).

$m R N A$ levels of IgG3 in the spleen correlates with the severity of GN. In our previous studies, it was found that some of the backcross mice between MRL/lpr and non-autoimmne $\mathrm{C} 3 \mathrm{H} / \mathrm{lpr}$ mice, $\mathrm{MRL} / \mathrm{lpr} \times(\mathrm{MRL} / \mathrm{lpr} \times \mathrm{C} 3 \mathrm{H} / \mathrm{lpr}) \mathrm{F}_{1}$, developed GN, possibly due to the segregation of the genetic backgroud responsible for GN in the MRL/lpr strain of mice (Nose et al. 1989). Taking advantage of the availability of these hybrid mice to examine the relationship between mRNA levels of IgG3 and GN, we determined mRNA levels of IgG3 and IgG2a in the spleen, and compared them with those of MRL/lpr, MRL/Mp- $+/+, \mathrm{C} 3 \mathrm{H} / \mathrm{lpr}$, and B6/lpr mice. The degree of expression of IgG3 mRNA was found to vary among these hybrid mice, and did not correlate with that of IgG2a mRNA (Fig. 4A). When the densitometric units of IgG3 mRNA were plotted against the index of glomerular lesions in each mouse, a high degree of positive correlation was found between the mRNA levels of IgG3 and the development of GN ( $R=0.79)$. However, no singnificant correlation was observed between those of IgG2a and the development of GN ( $R=0.29)$ (Fig. 4B). These results indicate that the production of IgG3 in MRL/lpr mice is one of the major factors responsible for the development of GN in these mice, and that this is a part of the genetic backgroud introduced into the backcross mice from the MRL/lpr strain. Moreover, it can be

Fig. 4. Correlation between the expression of IgG3 or IgG2a mRNA in the spleen and index of glomerular lesions in $\mathrm{MRL} / \mathrm{lpr} \times(\mathrm{MRL} / \mathrm{lpr} \times \mathrm{C} 3 \mathrm{H} / \mathrm{lpr}) \mathrm{F}_{1}$ mice (Takahashi et al. 1991). A: Dot blot analysis of the expression of IgG3 and IgG2a mRNA in several strains of 20 -wk-old mice. Serially diluted samples $(9,3$, and $1 \mu \mathrm{g})$ from total spleen RNA of each mouse was applied on a nitrocellulose membrane. The IgG3 probe used was the cDNA of the $\mathrm{H}$ chain $\mathrm{C}$ region of IgG3 prepared by the PCR method from the IgG3-producing hybridoma clone (1G3) established from MRL/lpr mice, which was specific for hybridoma RNA from the IgG3 clone, but not reacted with RNA from an IgG2a-producing clone, Hyl.2, or that from an IgG2b-producing clone, GORK. The IgG2a probe was prepared from the 498-bp Pst $\mathrm{I}-P s t \mathrm{I}$ fragment of germline $\mathrm{C}_{\gamma} 2 \mathrm{a}$ including the Hinge-CH2 domain. $\mathrm{M} / 1$, $\mathrm{MRL} / \mathrm{lpr}$ (the first lane, 6 wk old); M/n, MRL/Mp-+/+; C/1, C3H/lpr; B/1, $\mathrm{B} 6 / \mathrm{lpr}$; no. 1 to no. $26, \mathrm{MRL} / \mathrm{lpr} \times(\mathrm{MRL} / \mathrm{lpr} \times \mathrm{C} 3 \mathrm{H} / \mathrm{lpr}) \mathrm{F}_{1}$. B : Correlation between the IgG3 or IgG2a mRNA levels and incidence of glomerular lesions. Relative amounts of each mRNA are represented as densitometric units based on the results in A. 26 individual mice were tested. $\bigcirc, \cdots \cdots, \operatorname{IgG3}, \mathrm{y}=0.43+0.061 \mathrm{x}$; $\bullet,-$, IgG2a, $y=0.86+0.028 x$. 
postulated that nephritogenic IgG3 antibodies must be present in MRL/lpr mice. Thus, we intended to establish nephritogenic IgG3 antibody producing hybridomas as follows.

\section{Nephritogenic monoclonal IgG3 antibodies and their immunoglobulin genes}

Establishment of hybridoma clones producing nephritogenic IgG3 antibodies. Twenty-one hybridoma clones which produced IgG3 antibodies were obtained by cell fusion between MRL/lpr spleen cells and NS-1 myeloma cells. Each clone $\left(1 \times 10^{7}\right.$ cells $/$ mouse) was injected to normal $(\mathrm{MRL} / \mathrm{lpr} \times \mathrm{BALB} / \mathrm{c}) \mathrm{F}_{1}$ mice. 10 20 days after the injection, five of the twenty-one clones induced glomerular lesions in all of the injected mice (Takahashi et al. 1993). The 2B11.3 and three other clones induced proliferative lesions in glomeruli (Fig. 5A), whereas the glomerular lesion induced by the clone 7B6.8 was characterized by hyaline thrombi with wire-loop formation (Fig. 5B) (Itoh et al. 1993). These five clones generated cryoglobulins both in vivo and in vitro. Sixteen other clones did not induce histologic abnormalities in any of the injected mice.

Both in the 2B11.3- and 7B6.8-injected mice, severe immune deposits in the glomeruli containing IgG and C3 were demonstrated by immunofluorescent staining.

In 2B11.3-injected mice, numerous Mac-2 positive macrophage infiltrates were seen in glomeruli, but they were less or rare in 7B6.8-injected mice any day after the hybridoma injection. We examined the time course of the increase of serum IgG3 levels in $(\mathrm{MRL} / \mathrm{lpr} \times \mathrm{BALB} / \mathrm{c}) \mathrm{F}_{1}$ mice injected with $2 \mathrm{~B} 11.3$ or $7 \mathrm{~B} 6.8$ clone by single radial immunodiffusion. Changes over time of IgG3 levels in sera obtained from the injected mice were almost identical for both $2 \mathrm{~B} 11.3$ and $7 \mathrm{~B} 6.8$

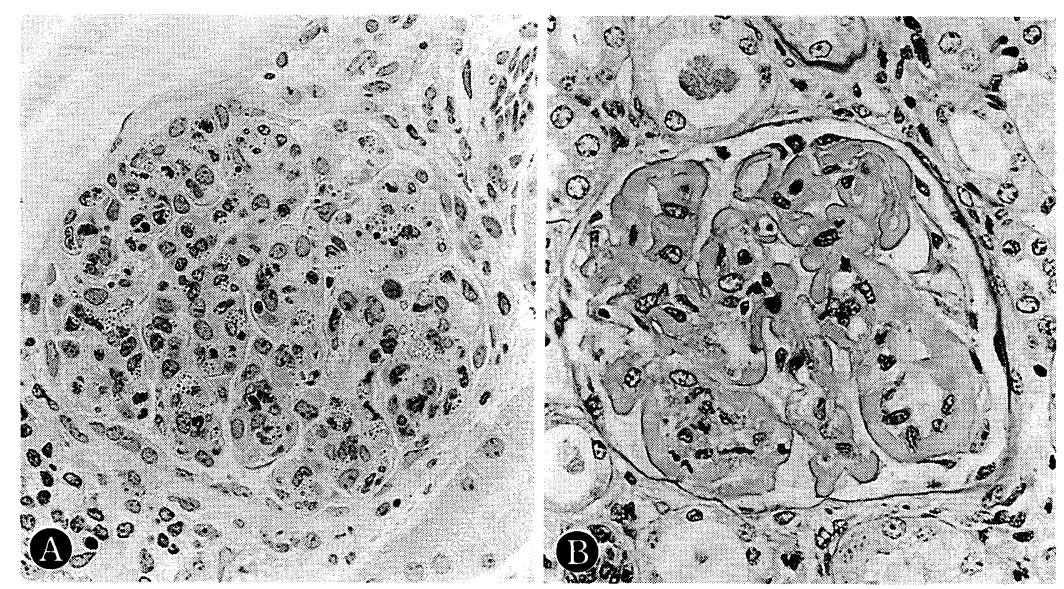

Fig. 5. Glomerular lesions induced by clone $2 \mathrm{~B} 11.3$ (A) or 7B6.8 (B), characterized by a marked infiltration of phagocytic cells (A), and by hyline thrombi and subendothelial deposits, resembling wire-loop lesions (B). 
clones. In addition, under an isoelectric foucussing analysis, purified 2B11.3 antibody molecules posessed slightly anionic charge (pI 6-6.2), but 7B6.8 antibody molecules were highly cationic (pI 8.3-8.6). Thus, we found that the antibodies themselves induce glomerular lesions and do so with different histopathological manifestations such as proliferative and wire-loop type GN. Moreover, our findings strongly suggest that the mechanisms for induction of glomerular injury are quite different for each of these two clones.

$c D N A$ cloning and sequence analyses of nephritogenic IgG3 antibodies. To examine whether the clones $2 \mathrm{~B} 11.3$ and $7 \mathrm{~B} 6.8$ originated from distinct $\mathrm{B}$ cell precursor, cDNA cloning and sequence analyses of these antibodies were performed (Takahashi et al. 1993). Their $\mathrm{V}$ region sequences revealed that these two IgG3 have different sets of $\mathrm{VH}, \mathrm{D}, \mathrm{JH}, \mathrm{V} \varkappa$ and $\mathrm{J}_{\varkappa}$ segments: $\mathrm{J} 558$, sp2., JH3, $\mathrm{V} \varkappa 4$ and $J_{\varkappa} 5$ for $2 B 11.3$, and J558, FL16.1, JH4, V $\varkappa 4$ and $\mathrm{J}_{\varkappa} 1$ for $7 \mathrm{~B} 6.8$ (Figs. 6 and 7). Moreover, nucleotide difference in $\mathrm{V}_{\mathrm{H}}$ segment between $2 \mathrm{~B} 11.3$ and $7 \mathrm{~B} 6.8$ were observed at 35 nucleotides (13 in FR1, 4 in CDR1, 2 in FR2, 10 in CDR2, and 6 in FR3) (Fig. 6). This result indicates that the $\mathrm{V}_{\mathrm{H}}$ germline genes of $2 \mathrm{~B} 11.3$ and 7B6.8 are actually different.

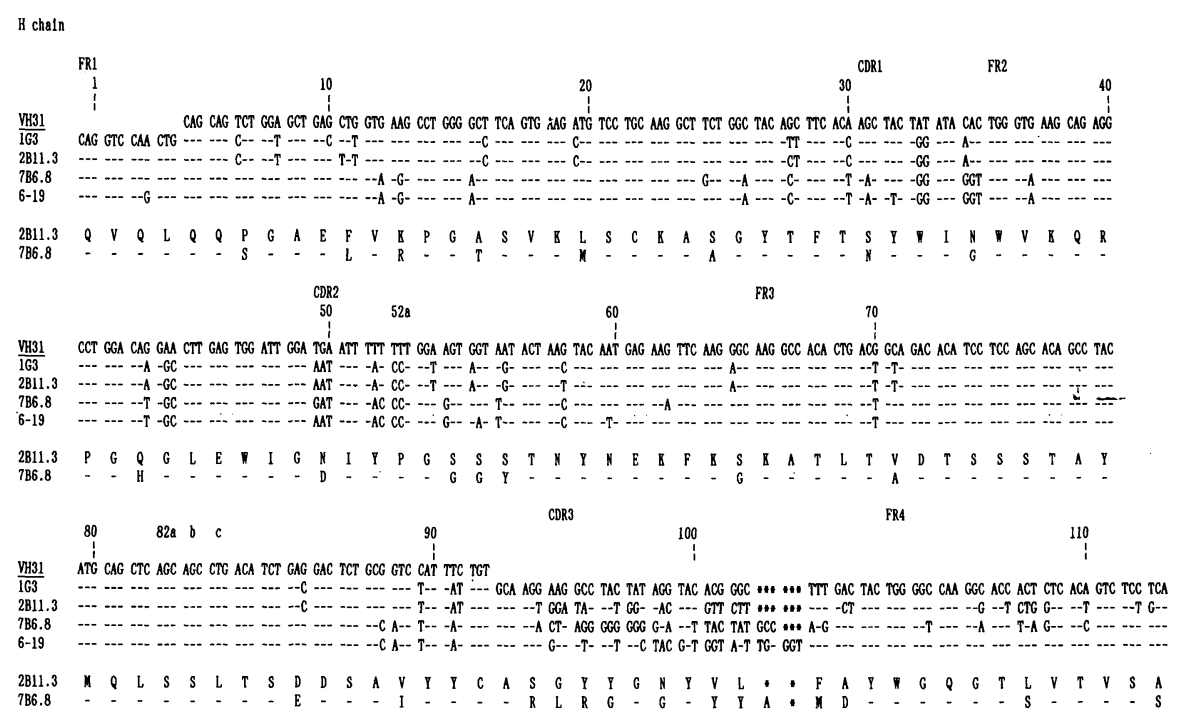

Fig. 6. Nucleotide and deduced amino acid sequences of the heavy chain $\mathrm{V}$ region of the nephritogenic and non-nephritogenic antibodies (Takahashi et al. 1993). 2B11.3, 7B6.8, and 6-19 (Reininger et al. 1990) are nephritogenic clones, while $1 \mathrm{G3}$ is nonnephritogenic. Numbering of amino acid residues and marking of FRs and CDRs are according to Kabat et al. (1987) Each nucleotide sequence was compared to the $\mathrm{V}_{\mathrm{H}} 31$ germline sequence derived from BALB/ $\mathrm{c}$ mice (Loh et al. 1983). Identities are indicated by dashes, and asterisks indicate gaps. 

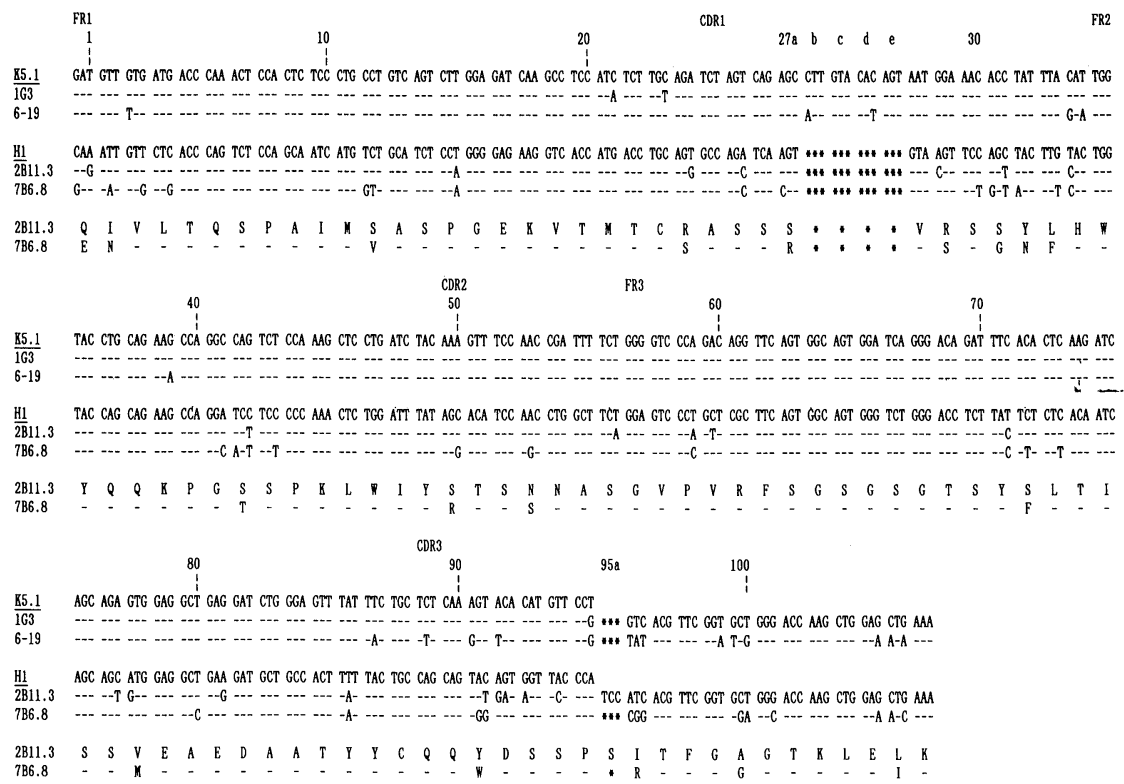

Fig. 7. Nucleotide and deduced amino acid sequences of the light chain variable region of the nephritogenic and non-nephritogenic antibodies (Takahashi et al. 1993). Each nucleotide sequence was compared to the germline sequences; $1 \mathrm{G} 3$ and 6-19 to $\mathrm{K} 5.1$ ( $\mathrm{V} \varkappa-1 \mathrm{~A}$ subgroup), and $2 \mathrm{~B} 11.3$ and $7 \mathrm{~B} 6.8$ to $\mathrm{H1}$, both of which are derived from BALB/c mice (Even et al. 1985; Corbet et al. 1987). Sequences are displayed as described in the legend to Fig. 6.

\section{Concluding remarks}

MRL/lpr mice spontaneously develop a lethal GN, resembling human lupus nephritis. IgG3 production in these mice was found to have a critical role in the development of GN: 1) IgG3 levels were high in kidney-extracted IgG and in circulating IgG IC, despite the predominance of IgG2a in sera, 2) serum IgG3, but not IgG2a, was reduced by CsA treatment, and 3) the mRNA levels of IgG3 in the spleen correlated with the severity of GN among the $\mathrm{MRL} / \mathrm{lpr} \times(\mathrm{MRL} / \mathrm{lpr} \times \mathrm{C} 3 \mathrm{H} /$ lpr) $\mathrm{F}_{1}$ mice with the rearranged genetic profile. Taking these findings into consideration, we attempted and succeeded in establishing five hybridomas which produce nephritogenic IgG3 antibodies from an MRL/lpr mouse. Four out of the five clones, e.g., clone 2B11.3, generated cell-proliferative GN, but the other clone 7B6.8 induced glomerular lesions characterized by subendothelial hyaline deposits resembling wire-loops. The cDNA sequence of the nephritogenic IgG3s revealed that the development of the two types of glomerular lesions were mediated by clonally different $B$ cell precursors. Thus, it was clear that particular IgG3 antibodies in MRL/lpr mice are nephritogenic at a monoclonal level. Moreover, our findings suggest that the histopathological variation in GN seen in MRL/lpr 
mice results from clonally expanded B cells that produce nephritogenic antibodies with different pathogenic potencies. This understanding may be applicable to investigations into human lupus nephritis.

\section{References}

1) Andrews, B.S., Eisenberg, R.A., Theofilopoulos, A.N. \& Dixon, F.J. (1978) Spontaneous murine lupus-like syndromes. Clinical and immunopathological manifestations in several strains. J. Exp. Med., 148, 1198-1215.

2) Corbet, S., Milili, M., Fougereau, M. \& Schiff, C. (1987) Two $V_{K}$ germ-line genes related to the GAT idiotypic network (Abl and Ab3/Abl) account for the major subfamilies of the mouse $\mathrm{V}_{\mathrm{K}}-1$ variability subgroup. J. Immunol., 138, 932-939.

3) Even, J., Griffiths, G.M., Berek, C. \& Milstein, C. (1985) Light chain germ-line genes and the immune response to 2-phenyl-oxalone. EMBO J., 4, 3439-3445.

4) Gavalchin, J. \& Datta, S.K. (1987) The NZB $\times$ SWR model of lupus nephritis I. Cross-reactive idiotypes of monoclonal anti-DNA antibodies in relation to antigenic specificity, charge, and allotype. Identification of interconnected idiotype families inherited from the normal SWR and the autoimmune NZB parents. J. Immunol., 138, 128-137.

5) Itoh, J., Nose, M. \& Kyogoku, M. (1991) Pathogenic significance of serum components in the development of autoimmune polyarthritis in MRL/lpr mice bearing the lymphoproliferation gene. Am. J. Pathol., 139, 511-521.

6) Itoh, J., Nose, M., Takahashi, S., Ono, M., Terasaki, S., Kondoh, E. \& Kyogoku, M. (1993) Induction of different types of glomerulonephritis by monoclonal antibodies derived from an MRL/lpr lupus mouse. Am. J. Pathol., 143, 1436-1443.

7) Izui, S., Elder, J.H., McConahey, P.J. \& Dixon, F.J. (1979) Association of circulating retroviral gp70-andi-gp70 immune complexes with murine systemic lupus erythematosus. J. Exp. Med., 149, 1099-1116.

8) Kabat, E.A., Wu, T.T., Reid-Miller, M., Perry, H.M. \& Gottesman, K.S. (1987) Sequences of Proteins of Immunological Interest. U.S. Depertment of Health and Human Services, NIH, Bethesda, M.D.

9) Loh, D.Y., Bothwell, A.L., White-Scharf, M.E., Imanishi-Kari, T. \& Baltimore, D. (1983) Molecular basis of a mouse strain-specific anti-hapten response. Cell, 33, 8593.

10) Mounts, J.D., Smith, H.R., Wilder, R.L., Reeves, J.P. \& Steinberg, A.D. (1987) Cs-A therapy of MRL-lpr/lpr mice: Amelioration of immunopathology despite autoantibody production. J. Immunol., 138, 157-163.

11) Nose, M., Nishimura, M. \& Kyogoku, M. (1989) Analysis of granulomatous arteritis on MRL/Mp autoimmune disease mice bearing lymphoproliferation gene. The use of mouse genetics to dissociate the development of arteritis and glomerulonephritis. Am. J. Pathol., 135, 271-280.

12) Reininger, L., Berney, T., Shibata, T., Spertini, F., Merino, R. \& Izui, S. (1990) Cryoglobulinemia induced by a murine IgG3 rhematoid factor: skin vasculitis and glomerulonephritis arise from distinct pathogenic mechanisms. Proc. Natl. Acad. Sci. USA, 87, 10038-10042.

13) Roberts, J.L., Wyatt, R.J., Schwartz, M.M. \& Lewis, E.J. (1983) Differential characteristics of immune-bound antibodies in diffuse proliferative and membranous forms of lupus glomerulonephritis. Clin. Immunol. Immunopathol., 29, 223-228.

14) Takahashi, S., Nose M., Sasaki, J., Yamamoto, T. \& Kyogoku, M. (1991) IgG3 production in MRL/lpr mice is responsible for development of lupus nephritis. $J$. Immunol., 147, 515-519.

15) Takahashi, S., Itoh, J., Nose, M., Yamamoto, T. \& Kyogoku, M. (1993) Cloning and 
sequence analysis of nephritogenic IgG3 monoclonal anticodies derived from an MRL/ lpr lupus mouse. Mol. Immunol., 30, 177-182.

16) Theofilopoulos, A.N. \& Dixon, F.J. (1985) Murine models of systemic lupus erythematosus. Adv. Immunol., 37, 269-280. 